\title{
The Anti-VEGF Antibody Bevacizumab Potently Reduces the Growth Rate of High-Risk Neuroblastoma Xenografts
}

\author{
LOVA SEGERSTRÖM, DIETER FUCHS, ULRIKA BÄCKMAN, KAJSA HOLMQUIST, ROLF CHRISTOFFERSON, \\ AND FARANAK AZARBAYJANI
}

\author{
Childhood Cancer Research Unit, Department of Women and Child Health, Karolinska Institutet, 17176 Stockholm, Sweden; Department \\ of Medical Cell Biology [L.S., D.F., U.B., K.H., R.C., F.A.], Uppsala University, 75123 Uppsala, Sweden; Department of Surgical \\ Sciences [R.C.], Section of Pediatric Surgery, Children's Hospital, Uppsala University, 75123 Uppsala, Sweden
}

\begin{abstract}
Neuroblastoma (NB) is a rapidly growing, wellvascularized childhood cancer that often presents with metastases. The overall five-year survival in NB is approximately $45 \%$ despite multimodality treatment, and therefore there is a clinical need for new therapeutic strategies. NB frequently overexpresses the angiogenic factor VEGF (vascular endothelial growth factor). The aim of this study was to investigate the effect of bevacizumab (Avastin $®$, Genentech/Roche), a humanized anti-VEGF-A antibody, on NB growth in three different xenograft models, chosen to resemble high-risk NB. The human NB cell lines SK-N-AS, IMR-32 and SH-SY5Y, which are poorly differentiated and overexpress VEGF-A, were injected s.c. in immunodeficient mice. Bevacizumab was given intraperitoneally twice weekly at $5 \mathrm{mg} / \mathrm{kg}$ body weight, starting at a tumor volume of $0.3 \mathrm{~mL}$. Bevacizumab significantly $(p<0.01-0.05)$ reduced NB growth in vivo without toxicity by causing a $30-63 \%$ reduction of angiogenesis, but had no effect on NB cell survival in vitro. Serum concentrations of VEGF-A increased two- to six-fold during bevacizumab therapy which did not result in faster tumor growth compared with control animals. Based on our experimental data we suggest consideration of bevacizumab in treatment of highrisk NB that does not respond to conventional therapy and that overexpresses VEGF. (Pediatr Res 60: 576-581, 2006)
\end{abstract}

$\mathrm{N}^{\mathrm{e}}$ euroblastoma (NB) is the most common extracranial solid tumor of childhood. It arises from sympathetic neuroectodermal cells and can occur in the abdomen, thorax and neck. Age over one year at diagnosis, advanced stage and unfavorable histology in resected tumors indicate a poor outcome. Other biologic markers of a poor outcome are loss of the short arm of chromosome one, more than three copies of the oncogene MYCN and di- and tetraploidy (1).

The survival of patients with high-risk NB is still poor despite aggressive protocols involving high-dose chemotherapy, total body irradiation and bone marrow transplantation, underlining the clinical need for more effective treatment. One new approach in cancer therapy is angiogenesis inhibition. In this approach, angiogenesis, the formation of new blood vessels from the endothelium of the existing vasculature, is

Received March 16, 2006; accepted June 10, 2006

L.S. and D.F. contributed equally to this work.

Correspondence: Faranak Azarbayjani, Ph.D., Department of Medical Cell Biology, Box 571, 75123 Uppsala, Sweden; e-mail: Faranak.Azarbayjani@mcb.uu.se

Supported by grants from the Swedish Cancer Society and the Children's Cancer Foundation of Sweden.

DOI: $10.1203 / 01 . p d r .0000242494 .94000 .52$ blocked, resulting in starvation of tumors and their metastases by blocking their nutritive blood supply (2). Specific inhibition of angiogenesis is considered to entail less toxicity than chemotherapeutics given at the maximally tolerated dose. Angiogenesis has been quantified in clinical NB, and is reported to be correlated to metastatic disease and to a poor outcome (3). VEGF-A is a potent angiogenic growth factor, and over-expression of VEGF-A has been observed in a variety of human cancers, including NB $(4,5)$. Recently it was shown that a specific inhibitor of angiogenesis, bevacizumab (Avastin®, Genentech/Roche; a humanized anti-VEGF-A antibody) prolongs survival and delays tumor progression in patients with metastatic colorectal cancer. Bevacizumab is now recommended as first-line therapy in combination with chemotherapy in these patients (6). Bevacizumab is thus the first clinically available specific inhibitor of angiogenesis.

The aim of this study was to investigate the effects of bevacizumab in three xenograft models of high-risk NB. We found that bevacizumab potently reduced NB growth most likely through inhibition of angiogenesis.

\section{MATERIALS AND METHODS}

Cell cultures. Three human neuroblastoma cell lines were chosen for xenografting: SK-N-AS (1p-deleted, derived from a 6-y-old girl with a poorly differentiated NB (7)), IMR-32 (1p-deleted, MYCN-amplified, derived from a 13-mo-old boy (8)) and SH-SY5Y (45 + XX karyotype due to trisomy of 1q and poorly differentiated neuroblastic phenotype, derived from a 4-y-old girl (9)). All three cell lines express VEGF-A and were obtained and cultured as described previously (10). Bovine capillary endothelial cells (BCE) were harvested and grown as described previously (11). All cultures were negative when tested for mycoplasms.

Fluorescent microculture cytotoxicity assay (FMCA). FMCA was used to determine whether bevacizumab was cytotoxic to NB cells or to endothelial cells. Cells were seeded at 20,000 or 5,000 cells per well, respectively, in 96-well plates (Nunc, Roskilde, Denmark). Bevacizumab, diluted in sterile PBS (SVA, Uppsala, Sweden), was added at concentrations ranging from $30-2,500 \mu \mathrm{g} / \mathrm{mL}$, four triplicates for each concentration, and incubated for $72 \mathrm{~h}$. Fluorescence was read at $528 \mu \mathrm{m}$ and the cell survival index was calculated as described earlier (12).

Abbreviations: BW, body weight; CgA, chromogranin A; FMCA, fluorescent microculture cytotoxicity assay; NB, neuroblastoma; RT, room temperature; TH, tyrosine hydroxylase 
Animals, xenografting, tumor measurement and perfusion fixation. $\mathrm{Fe}-$ male NMRI nu/nu mice (M \& B, Ry, Denmark) were xenografted at the age of $6 \mathrm{wk}$ (BW 18-24 g). The animals were housed in an isolated room at $24^{\circ} \mathrm{C}$ with a 12-h day/night cycle and fed ad libitum with water and food pellets. The animal weight and general appearance were recorded every other day throughout the experiments. The experiments were approved by the regional ethics committee for animal research.

Xenografting, tumor measurement and calculation of tumor size were performed as previously described (10). In short, for xenografting the mice were anesthetized with $2 \%$ fluothane (Zeneca Ltd, Macclesfield, UK) supplemented with $50 \% \mathrm{~N}_{2} \mathrm{O}$ in oxygen. $\mathrm{NB}$ cells $\left(30 \times 10^{6}\right.$ cells in $0.1 \mathrm{~mL}$ medium) were implanted s.c. in the right hindleg of the animal, using a $23 \mathrm{G}$ needle.

Tumors were measured every other day with a digital caliper, starting when they were palpable $(0.1 \mathrm{~mL})$. At a tumor volume of $0.3 \mathrm{~mL}$ the animals were randomized and treatment started. Bevacizumab (Genentech, South San Francisco, CA) was diluted in sterile PBS (SVA) and administered at $5 \mathrm{mg} / \mathrm{kg}$ BW IP twice weekly. Controls were given sterile PBS IP twice weekly. The experiments were terminated before the control tumors reached a volume of $4 \mathrm{~mL}$. No animals had to be killed before completion of any experimental endpoint. Perfusion fixation and autopsy were performed as described previously (13). Tumors were excised and tumor weight recorded. Internal organs were examined for macroscopic metastases to confirm the nonmetastatic behavior of the model.

Blood analyses. Blood was drawn from the right ventricle immediately before perfusion fixation, allowed to clot, stored at $4^{\circ} \mathrm{C}$ overnight and spun at $130 \times \mathrm{g}$ for $10 \mathrm{~min}$. The serum was removed and stored at $-70^{\circ} \mathrm{C}$.

For Western blot analyses, to demonstrate bevacizumab and VEGF in serum, samples were boiled for $5 \mathrm{~min}$, separated on 10\% SDS/PAGE, electrically blotted to nitrocellulose membrane and blocked with 5\% nonfat milk in PBS and $0.1 \%$ Tween-20. For detection of bevacizumab, the membrane was incubated with a polyclonal rabbit anti-human $\mathrm{IgG}_{1}$ antibody (P0212, DakoCytomation, Denver, CO) directly conjugated to horseradish peroxidase. Human VEGF-A was detected using a monoclonal anti-human VEGF antibody (MAB293; R \& D Systems, Abingdon, UK) followed by a horseradish peroxidase-conjugated secondary antibody (P0161, DakoCytomation, Denver, CO). Enhanced chemoluminescence (Amersham Pharmacia Biotech, Piscataway, NJ) was used for detection according to the manufacturer's instructions.

To measure VEGF-A levels in serum, samples were analyzed using a commercially available ELISA kit specific for human VEGF-A (DVE00, R\&D Systems, Minneapolis, MN) according to the manufacturer's recommendations.

Immunohistochemistry. Perfusion-fixed tumors were immersion-fixed in $4 \%$ paraformaldehyde before dehydration and paraffin embedding. Tissue sections were cut at $3 \mu \mathrm{m}$ and put on diaminoalkyl silane-treated glass slides
(Sigma Chemical Co. Chemical Co, St. Louis, MO). Sections were de-waxed, rehydrated and blocked in $0.3-3 \% \mathrm{H}_{2} \mathrm{O}_{2}$ in PBS for $30 \mathrm{~min}$ at room temperature (RT).

Bandeirea simplicifolia-1 (BS-1) lectin was used for highlighting murine endothelial cells as described previously (10). Biotinylated BS-1 (L3759, Sigma Chemical Co.) was diluted 1:50 and incubated either overnight at $8^{\circ} \mathrm{C}$ or for $1 \mathrm{~h}$ at RT, both methods staining equally.

Cell proliferation was detected by Ki-67 nuclear antigen staining as described previously (13).

Staining specific for neuroendocrine and adrenergic cells, i.e., NB cells, was performed by chromogranin $\mathrm{A}(\mathrm{CgA})$ and tyrosine hydroxylase $(\mathrm{TH})$ immunohistochemistry as described previously (13).

Apoptosis was detected by cleaved caspase-3 using a primary antibody (\#9661, affinity purified polyclonal anti-cleaved caspase-3, Cell Signaling Technology, Beverly, MA) and a secondary, horseradish peroxidase (HRP)conjugated antibody (\#7074, Cell Signaling Technology, Beverly, MA) according to the manufacturer's instructions.

Human pancreas, human or bovine adrenal gland cortex, human colonic mucosa, human breast carcinoma and endothelial cell pellets were used as positive controls for $\mathrm{CgA}$, TH, caspase-3, Ki-67 and BS-1 respectively. In all cases omission of primary antibodies served as a negative control.

The immunoreactivity for $\mathrm{CgA}, \mathrm{TH}, \mathrm{Ki}-67$ and $\mathrm{BS}-1$ was detected using ABComplex conjugated to HRP (K355, Dako A/S, Glostrup, Denmark), diluted $(1: 100)$ and incubated for $30 \mathrm{~min}$ at RT. Sections were developed using diaminobenzidine tetrahydrochloride (DAB) (SK-4100, Vector Laboratories Inc., Burlingame, CA), counterstained with Harris' hematoxylin and mounted with Kaiser's glycerol gelatin (Merck). All steps were performed in humidified chambers.

Stereological quantifications. All sections were quantified at $\times 400$ magnification and in a blinded fashion as described earlier (14).

Statistical methods. All data were processed in Statistica 5.1 for Windows (StatSoft Inc., Tulsa, OK). Differences between groups were tested with the Mann-Whitney U-test. Statistical significance of differences was defined as $p$ $<0.05$.

\section{RESULTS}

FMCA. Bevacizumab at concentrations ranging from $30-$ $2,500 \mu \mathrm{g} / \mathrm{mL}$ had no significant effect on the proliferation or survival of SK-N-AS, IMR-32, SH-SY5Y or BCE (data not shown).

Neuroblastoma growth. The tumor growth rate was reduced in all bevacizumab treated groups compared with con-

Table 1. Quantification of tumor dynamics

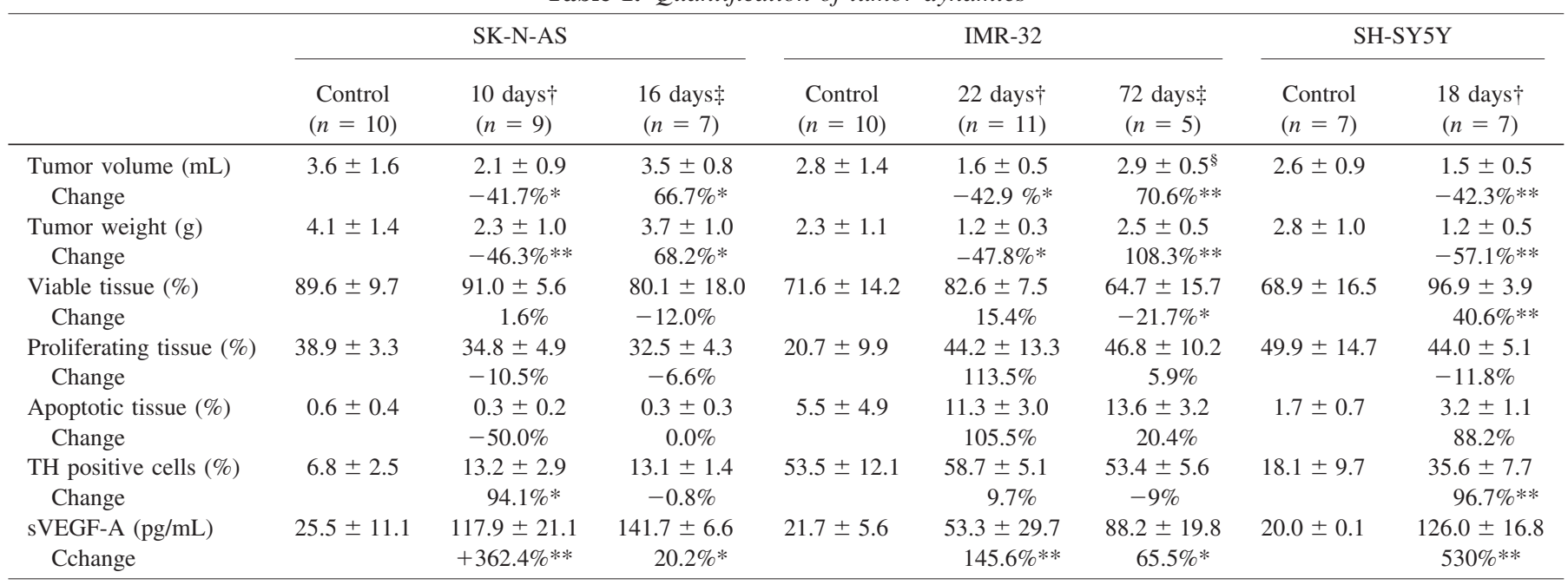

Different tumor parameters of three human neuroblastoma xenografts after IP treatment with $5 \mathrm{mg} / \mathrm{kg}$ bevacizumab twice weekly, presented as mean $\pm 1 \mathrm{SD}$.

Mann-Whitney U-test. The fraction of proliferating, apoptotic, and TH positive cells are given in percent of 2,000 counted cells per tumor.

$\mathrm{TH}$, tyrosine hydroxylase; sVEGF-A, serum vascular endothelial growth factor A.

$* p<0.05 ; * * p<0.01$.

$\dagger$ Change vs. control treatment; $\ddagger$ Change vs. short time treatment; $\S$ at day 56 of treatment. 

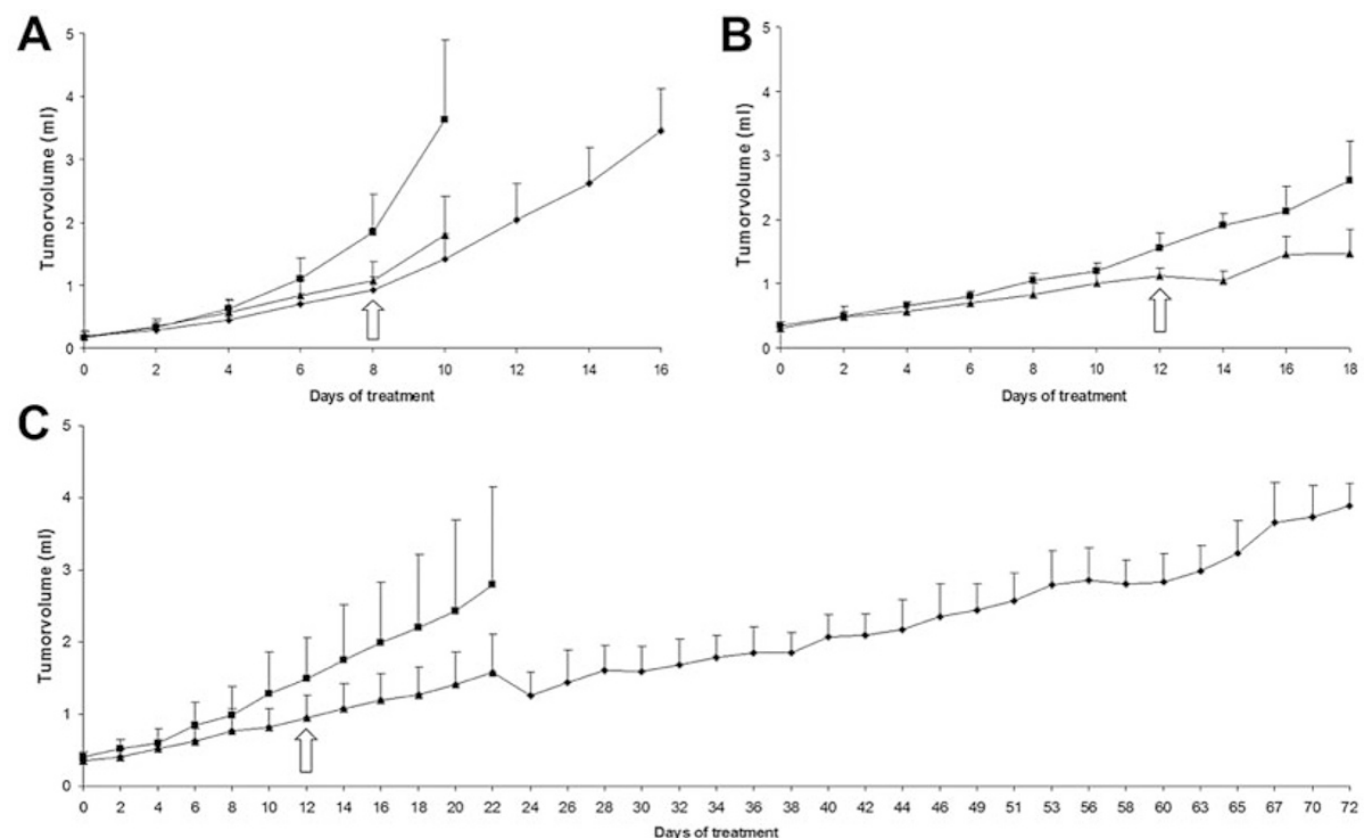

Figure 1. Subcutaneous neuroblastoma growth in nude mice. (A) SK-N-AS: controls $(\boldsymbol{\square} ; n=10)$, bevacizumab, $10 \mathrm{~d}(\mathbf{\Lambda} ; n=9)$ and $16 \mathrm{~d}$ of treatment $(\bullet ; n$ $=7)$. (B) SH-SY5Y: controls $(\boldsymbol{\square} ; n=7)$, bevacizumab, $18 \mathrm{~d}$ of treatment $(\boldsymbol{\Delta} ; n=7)$. (C) IMR-32: controls $(\mathbf{\square} ; n=10)$, bevacizumab, $22 \mathrm{~d}(\mathbf{\Delta} ; n=11)$ and $72 \mathrm{~d}$ of treatment $(\bullet ; n=5)$. Bevacizumab was given at $5 \mathrm{mg} / \mathrm{kg}$ BW IP twice weekly. Arrows indicate the day when groups first differed significantly. Results are expressed as mean +1 SEM. $p<0.05$; Mann-Whitney U-test.

trols, without any apparent toxicity. The difference between the groups became statistically significant at treatment day 8 for SK-N-AS and at day 12 for IMR-32 and SH-SY5Y (Table 1, Fig. 1). Prolonged treatment with bevacizumab (16 d for SK-N-AS and $72 \mathrm{~d}$ for IMR-32), delayed tumor progression for $6 \mathrm{~d}$ (SK-N-AS) and $31 \mathrm{~d}$ (IMR-32; Fig. 1). Administration of bevacizumab to nontumor bearing mice had no effect on body weight (BW) compared with naïve, untreated mice. The weight gain was similar in the control and bevacizumab treated group. At termination, the mean tumor volume in mice treated with bevacizumab divided by that in controls (T/C) was 0.58 in SK-N-AS, 0.57 in IMR-32 and 0.58 in SH-SY5Y. Control tumors were larger, softer and darker than bevacizumab treated tumors (Fig. 2). No tumor regression was seen.

Angiogenesis. Stereological quantifications of intratumoral blood vessels showed a decrease in vascular parameters in all three groups, reaching significance in SH-SY5Y (Table 2). As shown in Table 2, after $18 \mathrm{~d}$ of bevacizumab treatment of SH-SY5Y tumors there were significant reductions in the volumetric density (63\%), the surface density $(47 \%)$, the mean vessel area (57\%), the mean vessel boundary length $(36 \%)$ and the mean vessel diameter (35\%) compared with the vehicle treated controls.

VEGF-A concentrations in serum. The serum concentrations of human VEGF-A were significantly increased during bevacizumab treatment compared with controls for all three cell lines: $362 \%$ for SK-N-AS, $145 \%$ for IMR-32 and 530\% for SH-SY5Y (Table 1). Tumor bearing, vehicle treated control animals exhibited significantly higher VEGF-A levels than naïve mice and mice without tumors receiving bevacizumab treatment, indicating that NB tumors produce VEGF-A.

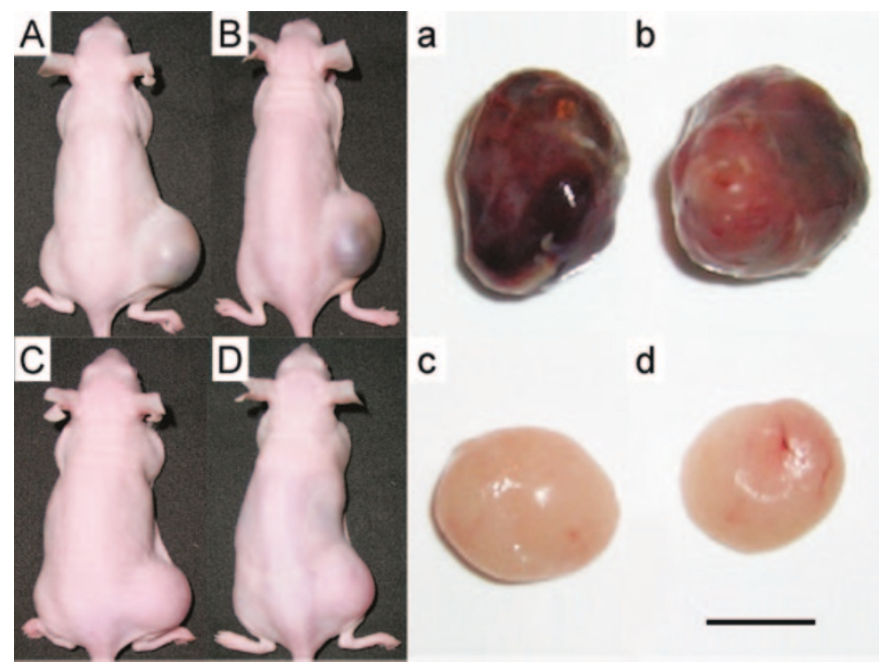

Figure 2. Representative mice with a s.c. SH-SY5Y neuroblastoma in the right hindleg $(A-D)$ and corresponding excised tumors $(a-d)$. Aa,Bbcontrols; $C c, D d-18 \mathrm{~d}$ of treatment with bevacizumab, $5 \mathrm{mg} / \mathrm{kg}$ BW, IP twice weekly. Bevacizumab treated tumors were flatter, more discoid, paler, and firmer than controls. Bar $=1 \mathrm{~cm}$.

The VEGF-A levels significantly increased additionally on prolonged treatment of SK-N-AS and IMR-32 xenografted animals. On Western blot analysis VEGF-A-immunoreactive material eluted at the same position as intact VEGF-A, indicating that VEGF-A is actively secreted by NB cells and is not released upon tumor cell lysis (data not shown). Western blot also confirmed the presence of human $\operatorname{IgG}$ (i.e. bevacizumab) in sera from treated animals but not from control animals (data not shown).

Tumor cell dynamics. The viable tissue fraction of tumors did not change significantly in SK-N-AS during the short or 
Table 2. Quantification of stereologic angiogenesis parameters

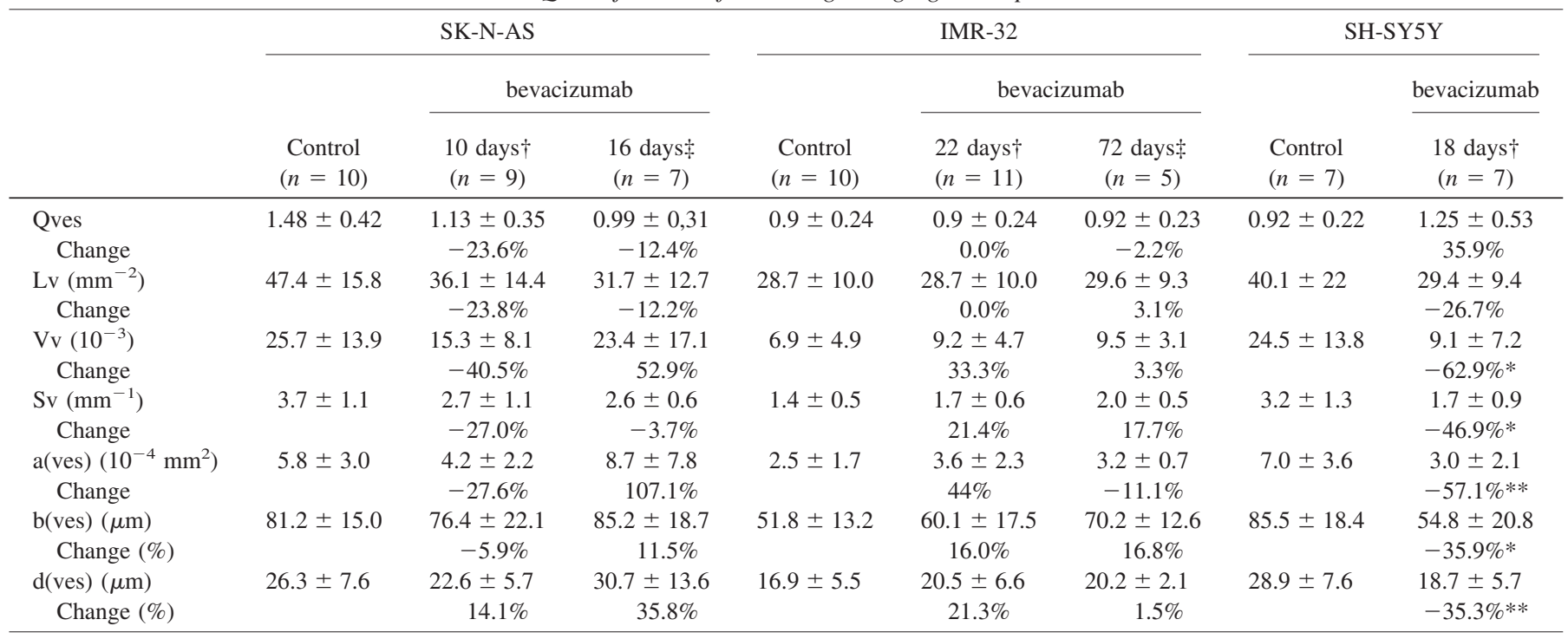

Bevacizumab treatment was $5 \mathrm{mg} / \mathrm{kg}$ twice weekly IP.

Qves, mean number of vessels per grid at $\times 400$; Lv, length of vessels per tumor volume (length density); Vv, volume of vessels per tumor volume (volumetric density); Sv, surface area of vessels per tumor volume (surface density); a(ves), mean section area of vessels; $b$ (ves), mean boundary length of vessels; $d(v e s)$, mean section diameter of vessels. Mean $\pm 1 \mathrm{SD}$, Mann-Whitney U-test.

$* p<0.05 ; * *<<0.01$.

$\dagger$ Change compared to control; $\ddagger$ Change compared to short time treatment.

prolonged treatment. In IMR-32 there was a significant reduction of the viable tissue fraction during prolonged treatment, while SH-SY5Y showed a significant increase in viable cell fraction upon treatment (Table 1). The fractions of proliferating and apoptotic cells did not change significantly for any of the three cell lines. Bevacizumab treated tumors displayed a sleeve like arrangement of neuroblastoma cells surrounding a central vessel, forming perivascular cuffs (Fig. 3A-D). In perivascular cuffs the proliferating cells were mainly located in the inner and middle layers, while apoptotic cells were confined to the outer layers (Fig. 3A,B). The tumors exhibited cells staining positively for $\mathrm{CgA}$ (Fig. $3 C$ ) and $\mathrm{TH}$ (Fig. $3 D$ ), confirming that the tumors were of NB origin. The fraction of TH positive cells were increased in all the three cell lines tested, reaching significant levels in SK-N-AS and SH-SY5Y xenografts (Table 1).

\section{DISCUSSION}

Bevacizumab as single therapy reduced tumor growth by $40 \%$ in our experimental models for high-risk NB without observable toxicity. Bevacizumab did not affect NB cell proliferation or survival in vitro, but induced a reduction of angiogenesis of up to $63 \%$ in vivo. Therefore, the effect of bevacizumab in NB is probably mediated through interference with tumor angiogenesis.

Over 30 angiogenesis inhibitors are currently in clinical trials in cancer patients (15). In a phase III clinical trial involving 813 patients with metastatic colorectal cancer, bevacizumab in combination with chemotherapy prolonged sur-

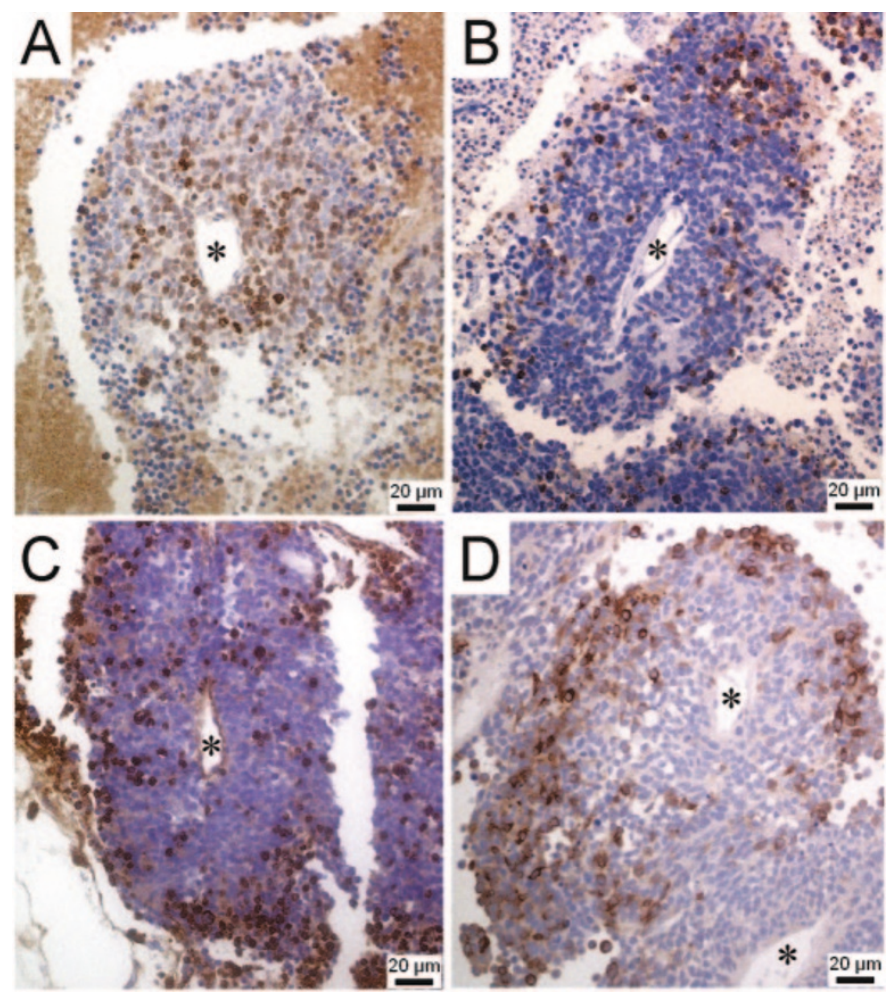

Figure 3. Representative morphology in neuroblastoma xenografts in animals treated with bevacizumab $(5 \mathrm{mg} / \mathrm{kg})$ for $72(A, C)$ or $18 \mathrm{~d}(B, D)$, respectively. Perivascular cuff of proliferating IMR-32 cells (Ki67 positive; brown) in proximity to a blood vessel $(*)(A)$. Perivasulcar cuff with apoptotic (Casp3 positive; brown) SH-SY5Y cells distant from a vessel $(*)(B)$. IMR-32 cells positive for $\mathrm{CgA}(C)$. Perivascular cuff of $\mathrm{TH}$ positive cells (brown) surrounding a central vessel in an SH-SY5Y tumor $(D)$. 
vival and delayed tumor progression compared with chemotherapy alone (6). Hypertension was the only side effect seen in these patients that was clearly related to the addition of bevacizumab to the standard chemotherapy regimen (6). There are preliminary reports of delayed tumor progression with addition of bevacizumab also in non-small-cell lung cancer and carcinoma of the breast and kidney. Bevacizumab is now approved as first-line therapy in advanced colorectal cancer in the U.S. and Europe, and is thus the first specific inhibitor of angiogenesis that is clinically available.

The reduction in tumor growth with bevacizumab in this study was lower than that after VEGF receptor blockade in our previous study (65\% growth reduction in SH-SY5Y (16)). There are three reasons why receptor blockade seems more efficient: First, bevacizumab does not block host derived murine VEGF, whereas an antibody blocking both human and murine-derived VEGF has shown to completely inhibit rhabdomyosarcoma growth in an experimental model (17). Second, bevacizumab is unlikely to inactivate matrix-bound VEGF-A and hence its associated paracrine activity is not abrogated. Third, receptor blockade may inhibit endocrine, paracrine and autocrine survival signals mediated through VEGFR 1 and 2 on NB cells. It can also be speculated that VEGF is one of several angiogenic growth factors in NB, since anti-VEGF antibodies cause a much more drastic effect in Wilm's tumor xenografts despite similar amounts of VEGF in these two tumors (18). With these limitations in mind, it must be stressed that bevacizumab is the first angiogenesis inhibitor that actually prolongs patient survival by working within the vasculature and it does not have to penetrate the tumor parenchyma to be pharmacologically active (19).

Serum VEGF-A concentrations rose during bevacizumab therapy in this study, a phenomenon which has also been observed in patients (20). This rise can in part be explained by reduced clearance of VEGF-A due to binding to bevacizumab $(20,21)$ or HIF- $1 \alpha$ mediated up-regulation of VEGF by increased hypoxia in NB cells (22). Evidently, the rise in serum VEGF was ineffectual as the control tumors grew faster than those of treated animals.

The dose of bevacizumab given in this study was identical to that in patients (6) but with a shorter interval, twice weekly compared with every second week. We do not expect that increasing the dose would increase the anti-tumor activity in view of the long plasma half-life of bevacizumab (20 d; Genentech, data on file). There are, however, experimental and clinical evidence of synergistic effects when an inhibitor of angiogenesis is combined with chemotherapy (23). Several studies have shown that low dose chemotherapy given more frequently (so-called metronomic dosing) can inhibit angiogenesis (24-26). The rationale for this combination therapy is "collateral damage," i.e. that low-dose cytotoxic drugs would damage the dividing endothelial cells in the newly formed tumor blood vessels and that the ability of these activated endothelial cells to cope with this damage is abrogated when not enough survival factors like VEGF-A are available (27). "Normalization" is another recently suggested hypothesis for the synergism observed when chemotherapy is combined with angiogenesis inhibitors. Based on this hypothesis, anti- angiogenic therapy, especially antibodies against VEGF-A such as bevacizumab, does not only reduce tumor angiogenesis but also normalizes the leaky tumor vessels and converts them back to a more normal status and thereby augments the delivery of chemotherapy and oxygen to the targeted cancer cells $(28,29)$. In fact, the combination of bevacizumab with chemotherapy has shown survival benefits in heavily treated colorectal and untreated, metastatic, breast cancer patients whereas combination of the small-molecule tyrosine kinase receptor inhibitor, vatalanib, with chemotherapy did not. One explanation might be that by inhibiting PDGFR- $\beta$ that is important in perivascular cell recruitment, vatalanib as well as other broad spectrum multitargeted tyrosine kinase inhibitors, interfere with vascular normalization thus preventing the synergistic effect of combined therapy (30).

In conclusion, by using well-documented experimental models for high-risk NB, we found that bevacizumab gave a $40 \%$ reduction of NB growth without systemic toxicity. We believe that bevacizumab inhibits angiogenesis by immunoneutralizing VEGF-A secreted by tumor cells and induces normalization of tumor vasculature. We expect to see increased anti-tumor activity if bevacizumab is combined with chemotherapy. On the basis of our experimental data, we suggest consideration of bevacizumab for adjuvant treatment of patients with high-risk NB that do not respond to conventional therapy and that overexpress VEGF.

Acknowledgments. Barbro Einarsson provided excellent technical assistance. Bevacizumab was provided by Genentech Inc (South San Francisco, CA). Serum VEGF analyses were kindly performed by Professor Anders Larsson at the Department of Clinical Chemistry at Akademiska Hospital, Uppsala.

\section{REFERENCES}

1. Schwab M, Westermann F, Hero B, Berthold F 2003 Neuroblastoma: biology and molecular and chromosomal pathology. Lancet Oncol 4:472-480

2. Folkman J 1971 Tumor angiogenesis: therapeutic implications. N Engl J Med 285:1182-1186

3. Meitar D, Crawford SE, Rademaker AW, Cohn SL 1996 Tumor angiogenesis correlates with metastatic disease, N-myc amplification, and poor outcome in human neuroblastoma. J Clin Oncol 14:405-414

4. Fakhari M, Pullirsch D, Paya K, Abraham D, Hofbauer R, Aharinejad S 2002 Upregulation of vascular endothelial growth factor receptors is associated with advanced neuroblastoma. J Pediatr Surg 37:582-587

5. Komuro H, Kaneko S, Kaneko M, Nakanishi Y 2001 Expression of angiogenic factors and tumor progression in human neuroblastoma. J Cancer Res Clin Oncol 127:739-743

6. Hurwitz HI, Fehrenbacher L, Hainsworth JD, Heim W, Berlin J, Holmgren E, Hambleton J, Novotny WF, Kabbinavar F 2005 Bevacizumab in combination with fluorouracil and leucovorin: an active regimen for first-line metastatic colorectal cancer. J Clin Oncol 23:3502-3508

7. Van Roy N, Jauch A, Van Gele M, Laureys G, Versteeg R, De Paepe A, Cremer T, Speleman F 1997 Comparative genomic hybridization analysis of human neuroblastomas: detection of distal $1 \mathrm{p}$ deletions and further molecular genetic characterization of neuroblastoma cell lines. Cancer Genet Cytogenet 97:135-142

8. Zaizen Y, Taniguchi S, Suita S 1998 The role of cellular motility in the invasion of human neuroblastoma cells with or without $\mathrm{N}$-myc amplification and expression. J Pediatr Surg 33:1765-1770

9. Biedler JL, Roffler-Tarlov S, Schachner M, Freedman LS 1978 Multiple neurotransmitter synthesis by human neuroblastoma cell lines and clones. Cancer Res 38:3751-3757

10. Backman U, Christofferson R 2005 The selective class III/V receptor tyrosine kinase inhibitor SU11657 inhibits tumor growth and angiogenesis in experimental neuroblastomas grown in mice. Pediatr Res 57:690-695 
11. Qi JH, Matsumoto T, Huang K, Olausson K, Christofferson R, Claesson-Welsh L 1999 Phosphoinositide 3 kinase is critical for survival, mitogenesis and migration but not for differentiation of endothelial cells. Angiogenesis 3:371-380

12. Larsson R, Kristensen J, Sandberg C, Nygren P 1992 Laboratory determination of chemotherapeutic drug resistance in tumor cells from patients with leukemia, using a fluorometric microculture cytotoxicity assay (FMCA). Int J Cancer 50:177-185

13. Svensson A, Backman U, Jonsson E, Larsson R, Christofferson R 2002 CHS 828 inhibits neuroblastoma growth in mice alone and in combination with antiangiogenic drugs. Pediatr Res 51:607-611

14. Wassberg E, Hedborg F, Skoldenberg E, Stridsberg M, Christofferson R 1999 Inhibition of angiogenesis induces chromaffin differentiation and apoptosis in neuroblastoma. Am J Pathol 154:395-403

15. Christofferson R, Claesson-Welsh L, Muhr C 2002 [Anti-angiogenic drugs probable complement in cancer therapy]. Lakartidningen 99:4138- 4139:4142-4138

16. Backman U, Svensson A, Christofferson R 2002 Importance of vascular endothelial growth factor $\mathrm{A}$ in the progression of experimental neuroblastoma. Angiogenesis 5:267-274

17. Gerber HP, Kowalski J, Sherman D, Eberhard DA, Ferrara N 2000 Complete inhibition of rhabdomyosarcoma xenograft growth and neovascularization requires blockade of both tumor and host vascular endothelial growth factor. Cancer Res 60:6253-6258

18. Kim E, Moore J, Huang J, Soffer S, Manley CA, O'Toole K, Middlesworth W, Stolar CJ, Kandel JJ, Yamashiro DJ 2001 All angiogenesis is not the same: Distinct patterns of response to antiangiogenic therapy in experimental neuroblastoma and Wilms tumor. J Pediatr Surg 36:287-290

19. Gerber HP, Ferrara N 2005 Pharmacology and pharmacodynamics of bevacizumab as monotherapy or in combination with cytotoxic therapy in preclinical studies. Cancer Res 65:671-680
20. Gordon MS, Margolin K, Talpaz M, Sledge GW Jr, Holmgren E, Benjamin R, Stalter S, Shak S, Adelman D 2001 Phase I safety and pharmacokinetic study of recombinant human anti-vascular endothelial growth factor in patients with advanced cancer. J Clin Oncol 19:843-850

21. Hsei V, Deguzman GG, Nixon A, Gaudreault J 2002 Complexation of VEGF with bevaczumab decreases VEGF clearance in rats. Pharm Res 19:1753-1756

22. Semenza GL 2003 Targeting HIF-1 for cancer therapy. Nat Rev Cancer 3:721732

23. Sweeney CJ, Miller KD, Sledge GW Jr 2003 Resistance in the anti-angiogenic era: nay-saying or a word of caution? Trends Mol Med 9:24-29

24. Belotti D, Vergani V, Drudis T, Borsotti P, Pitelli MR, Viale G, Giavazzi R, Taraboletti G 1996 The microtubule-affecting drug paclitaxel has antiangiogenic activity. Clin Cancer Res 2:1843-1849

25. Vacca A, Iurlaro M, Ribatti D, Minischetti M, Nico B, Ria R, Pellegrino A, Dammacco F 1999 Antiangiogenesis is produced by nontoxic doses of vinblastine. Blood 94:4143-4155

26. Clements MK, Jones CB, Cumming M, Daoud SS 1999 Antiangiogenic potential of camptothecin and topotecan. Cancer Chemother Pharmacol 44:411-416

27. Kerbel RS, Viloria-Petit A, Klement G, Rak J 2000 'Accidental' anti-angiogenic drugs. anti-oncogene directed signal transduction inhibitors and conventional chemotherapeutic agents as examples. Eur J Cancer 36:1248-1257

28. Jain RK 2005 Normalization of tumor vasculature: an emerging concept in antiangiogenic therapy. Science 307:58-62

29. Jain RK, Duda DG, Clark JW, Loeffler JS 2006 Lessons from phase III clinical trials on anti-VEGF therapy for cancer. Nat Clin Pract Oncol 3:24-40

30. Jain RK 2003 Molecular regulation of vessel maturation. Nat Med 9:685-693 